\title{
Assessment of cognition using the Rao's Brief Repeatable Battery of Neuropsychological Tests on a group of Brazilian patients with multiple sclerosis
}

\author{
Joseph Bruno Bidin Brooks ${ }^{1}$, Mariana Cossi Monseff Borela², \\ Yara Dadalti Fragoso ${ }^{3}$
}

\begin{abstract}
To assess the cognition of patients with multiple sclerosis (MS) using the Rao's Brief Repeatable Battery of Neuropsychological Tests (BRB-N). Method: BRB-N was translated and adapted for control subjects. Subsequently, it was applied to a group of patients with relapsing-remitting (RR) MS. Results: The assessment on the healthy controls ( $n=47$ ) showed that the correlation between tests on the same cognitive domain was high and that there was a five-factor solution that explained $90 \%$ of the total variance. Except for the Word List Generation subset of tests, the performance of patients with RRMS $(n=39)$ was worse than that of the healthy controls. Conclusion: BRB-N is a relatively simple method to assess cognition of patients with MS in the daily clinic. It does not take long to apply and does not require special skills or equipment.
\end{abstract}

Key words: multiple sclerosis, cognition, memory, neuropsychology.

Avaliação da cognição usando a Bateria Breve de Testes Neuropsicológicos de Rao para pacientes brasileiros com esclerose múltipla

\section{RESUMO}

Avaliar a cognição de pacientes com esclerose múltipla (EM), utilizando a Bateria Breve de Testes Neuropsicológicos de Rao (BRB-N). Método: BRB-N foi traduzida e adaptada para controles. Subsequentemente, foi aplicada em um grupo de pacientes portadores de EM RR. Resultados: A avaliação de controles saudáveis $(n=47)$ mostrou que a correlação entre os testes de um mesmo domínio cognitivo era alta e que uma solução de fator cinco explicava $90 \%$ da variação total. Exceto pela Lista de Palavras, o desempenho dos pacientes com EMRR $(n=39)$ foi pior que aquele dos controles saudáveis. Conclusão: BRB-N é um método relativamente simples de avaliar cognição de pacientes com EM na prática clínica. $O$ teste não requer muito tempo e não necessita de treinamento ou equipamentos especiais.

Palavras-Chave: esclerose múltipla, cognição, memória, neuropsicologia.

\section{Correspondence}

Joseph Bruno Bidin Brooks

Departments of Physiology and Neurology Medical School - UNIMES

Rua da Constituição 374

11015-470 Santos SP - Brasil

E-mail: joseph3b@gmail.com

Received 1 March 2011

Received in final form 15 June 2011

Accepted 20 June 2011
Cognitive dysfunction is increasingly recognized as a critical factor in the quality of life of patients with multiple sclerosis $(\mathrm{MS})^{1,2}$. Although more frequent in patients with brain atrophy and longduration disease, cognitive dysfunction may affect $20 \%$ of MS patients with mild disability in the earlier stages of the disease $^{3}$. From the clinical point of view, the functional status of patients with MS differs according to whether they have cognitive dysfunction or not ${ }^{4}$. Neuropsycho- 
logical rehabilitation of MS patients is challenging, since several aspects of the disease (fatigue, depression, pain, among others) may influence both the assessment and the rehabilitation of cognition ${ }^{5}$.

Brain lesions and atrophy seem to be the anatomical substrate for the clinical cognitive findings ${ }^{6,7}$, although intellectual enrichment seems to positively affect cognition despite the severity of brain lesions ${ }^{8}$. A recent review from Tiemann et al. ${ }^{9}$ highlighted the ongoing developments on the subject of brain lesion location and cognitive impairment. Notwithstanding all the recent scientific progress, the subject of cognitive dysfunction in MS remains open to discussion. For many authors, one of the key points of discussion on this subject is the instrument for cognitive assessment ${ }^{10}$. While a full neuropsychological evaluation is, indisputably the gold standard approach to assessing cognition in MS, few are the groups who can count on a neuropsychologist to perform tests in all patients. Suspected cases are usually referred for full testing, but suspicion may arise only at later stages of the disease, thus negatively influencing the results of cognitive rehabilitation. It is important to have scales or tests that could easily be applied during a routine consultation and thereby enable early detection of cases needing full investigation.

There are several scales and test batteries for assessing cognition in $\mathrm{MS}^{11}$. None of them is perfect ${ }^{12}$, and comparative studies among different batteries of tests show the Brief Repeatable Battery of Neuropsychological Tests (BRB-N) to be reliable and sensitive ${ }^{13}$. BRB-N was developed as a short and sensitive test to identify disturbances of cognitive domains in MS patients ${ }^{14}$. For daily practice as a screening method for non-neuropsychologists, the BRB-N could prove useful in cases of MS. A recent study by Portaccio et al. ${ }^{15}$ showed that, with testing lasting five to 15 minutes, cognitive impairment could be detected with the BRB-N with $94 \%$ sensitivity, $84 \%$ specificity, and $89 \%$ accuracy.

Despite the ease of use of BRB-N, there is a lack of Brazilian studies using this method for assessing MS patients' cognition. The aim of the present work was to translate, adapt and perform the BRB-N assessment on Brazilian patients with MS, and to discuss the findings in the light of similar reports from other countries.

\section{METHOD}

This study was approved by the Ethics Committee of Universidade Metropolitana de Santos, Santos SP, Brazil, and all participants were aware of the experimental nature of the study. They all signed an informed consent statement to take part in the evaluations. Prior to BRB-N testing, all subjects participating in the study underwent the Brazilian version of the mini-mental state examina- tion (MMSE) ${ }^{16}$. This was done in order to exclude the very severe cases of dementia, which were not to be assessed at the stage of our study.

\section{Translation and adaptation of the BRB-N}

The tests were translated by a medical doctor and retranslated by another doctor. Both doctors involved in this phase of the study were fully proficient and very experienced in the English language, using it on a daily basis.

The re-translated version of the tests was then sent to a certified medical translator for Portuguese to English, who was an English native-speaker.

The final format of the test presentation was then applied to a cohort of individuals with no neurological diseases, except for a few cases of primary, episodic headaches.

\section{Application to healthy individuals}

In order to obtain normative values for later comparison with MS patients, the translated version of BRB-N was applied to younger individuals than is usually done when it is translated and adapted. Furthermore, apart from claiming to be in good health, well rested and calm, these individuals answered the hospital anxiety/depression (HAD) questionnaire ${ }^{17}$, in order to avoid the negative influence of these conditions on the cognition assessment.

All participants in this phase of the study reported that they fully understood the tests.

\section{Application to MS patients}

MS patients regularly attending consultations in our MS unit were invited to participate in the study. Only patients with the clinical presentation of relapsing-remitting (RR) $M S^{18}$ were included in the study. Disease duration, disability (assessed by the expanded disability status scale; EDSS ${ }^{19}$ ) or mild to moderate fatigue were not considered in the exclusion criteria. However, patients scoring moderate to severe anxiety and/or depression in HAD were not included in this phase of the study. Patients who had presented a recent (less than 30 days) relapse of the disease were also excluded from testing. Like the healthy control individuals, MS patients claimed to have slept well the night before and that they felt calm at the testing evaluation.

\section{Testing}

The BRB-N includes: the Symbol Digit Modalities Test (SDMT) to assess attention, visual precision and executive functions; the Selective Reminding Test (SRT) to assess verbal memory and delayed recall; the 10/36 Spatial Recall Test to assess visual-spatial memory and delayed recall; and the Word List Generation (WLG) to assess semantic retrieval and verbal fluency. 
Three criteria were used for obtaining cut-off values: 1 standard deviation (SD), 1.5 SD and 2 SD below the mean values of the health controls in each test.

\section{RESULTS}

The demographic and clinical characteristics of the healthy controls and MS subjects are shown in Table 1. Both groups were homogeneously distributed in terms of age and education. A summary of results from both groups is shown in Table 2.

\section{Results from the assessment on the healthy controls}

The assessment on the 47 healthy controls showed that the correlation between tests on the same cognitive domain was high ( $r=0.6$; between the Selective Reminding Test and PASAT 2 or 3 PASAT 2). In the healthy subjects, there was a five-factor solution that explained $90 \%$ of the total variance, associated with the results from both the Selective Reminding Test and the Symbol Digit Modalities Test.

Age influenced the subjects' performance in the Selective Reminding Test (three subtests) and the Symbol Digit Modalities Test $(\mathrm{p}<0.05$ in all cases), while no differences in the performance of these individuals were observed with respect to gender. Education influenced the subjects' performance in the Symbol Digit Modalities Test, Symbol Digit Modalities Test, PASAT 2-3 and the Word List Generation tests ( $\mathrm{p}<0.05$ in all cases).

In summary, when control subjects were tested, they showed good correlation of results for tests of the same domain. Age and education significantly influenced the results.

\section{Results from the assessment on the MS patients}

The performance of the 39 RRMS patients in the BRB-N was worse than that of the healthy controls, except for the Word List Generation subset of tests. These data are shown in Table 2.

The BRB-N cut-off scores were stratified by age and three different education level criteria (Table 1). Using these cut-off levels, cognitive impairment was highly prevalent in MS patients. Using the least strict criterion, which would be only $1 \mathrm{SD}$ in one subtest, there was a very high prevalence of cognitive dysfunction (89.83\%) in the MS group, albeit with a large overlap with the healthy controls, since $64.47 \%$ of the controls had one abnormal subtest with this criterion.

When the criteria were set to medium stringency for abnormal performance (1.5 SD) in two subtests), the prevalence fell to around $60 \%$ for MS patients $(14.47 \%$ in healthy controls). However, even when the most stringent criteria were applied, such as an abnormal result (2 $\mathrm{SD}$ ) in three tests, cognitive impairment was still very prevalent in the MS patients (30\%), while this finding was present only in $3.94 \%$ of the healthy controls.

The results from BRB-N and the patients' physical disabilities showed a moderate correlation in all subtests, except for the Word List Generation test $(\mathrm{r}=0.395$ to $0.598, \mathrm{p}<0.05$ for EDSS; and $\mathrm{r}=0.276$ to $0.477, \mathrm{p}<0.05$ for MS Functional Composite; MSFC). The Symbol Digit Modalities Test showed a very strong correlation with both the EDSS ( $\mathrm{r}=0.598 ; \mathrm{p}<0.001)$ and the MSFC $(r=0.477 ; \mathrm{p}<0.001)$. This association between the Symbol Digit Modalities Test and the disability scales did not seem be due to hand motor impairment, since an independent association was maintained after adjusting for the dominance of the 9-HPT score $(r=0.610, p<0.001$ for

Table 1. Demographic and clinical characteristics of the healthy controls and MS subjects undergoing BRB-N testing.

\begin{tabular}{lcc}
\hline Clinical data & MS patients & Control group \\
\hline Number of participants & 39 & 47 \\
Gender (male/female) & $18-21$ & $22-25$ \\
Average age & $40.1( \pm 11.1)$ & $40.7( \pm 10.6)$ \\
$<35$ years & 11 & 13 \\
35-49 years & 19 & 24 \\
$>49$ years & 9 & 10 \\
EDSS & $2.71(0-7.0)$ & Not applicable \\
0-3.0 & $2.0(34-44)$ & \\
3.5-6.0 & $3.5(4-44)$ & \\
6.5-9.0 & $7.0(1-44)$ & \\
Time since diagnosis (years) & $2.2(0.4-8)$ & Not applicable \\
Average number of relapses & $2.5(1-8)$ & Not applicable \\
\hline
\end{tabular}

MS: multiple sclerosis; EDSS: expanded disability status scale. BRB-N: Brief Repeatable Battery of Neuropsychological Tests.

Table 2. Summary of results from cognition testing for controls and for patients with MS.

\begin{tabular}{lcc}
\hline Mini-mental state exam & MS patients & Control group \\
\hline Spatial orientation & $4.95(4-5) 93 \%$ & $4.97(4-5) 99 \%$ \\
Temporal orientation & $4.86(4-5) 97.2 \%$ & $4.90(4-5) 98 \%$ \\
Immediate memory & $3.0(3-3) 100 \%$ & $3.0(3-3) 100 \%$ \\
Attention and calculations & $3.50(1-5) 70 \%$ & $4.60(4-5) 92 \%$ \\
Evoked memory & $2.70(1-3) 90 \%$ & $2.90(1-3) 96.6 \%$ \\
Naming & $2.0(2-2) 100 \%$ & $2.0(2-2) 100 \%$ \\
Repetition & $1.0(1-1) 100 \%$ & $1.0(1-1) 100 \%$ \\
Verbal command & $1.0(1-1) 100 \%$ & $1.0(1-1) 100 \%$ \\
Motor command & $2.81(2-3) 93.3 \%$ & $2.9(2-3) 96.6 \%$ \\
Sentence & $1.0(1-1) 100 \%$ & $1.0(1-1) 100 \%$ \\
Drawing & $0.8(0-1) 80 \%$ & $0.95(0-1) 95 \%$ \\
Total & $27.77(24-30) 93 \%$ & $28.4(26-30) 94.7 \%$ \\
\hline MS: $m u$
\end{tabular}

MS: multiple sclerosis. 
EDSS; and $\mathrm{r}=0.469, \mathrm{p}<0.001$ for MSFC). According to the categories of the EDSS scale, patients with low disability (EDSS: 0 to 3.0) performed better than patients with medium disability (EDSS: 3.5 to 6.5 ) and highly disabled patients (EDSS: 7.0 to 9.0) in the Selective Reminding Test, the Symbol Digit Modalities Test and PASAT 2 and 3 tests $(\mathrm{p}<0.05$ in all cases).

Interestingly, MS patients with medium and high disability performed similarly in all tests. In addition, the performance of patients with very low disability (EDSS zero to 1.5) was similar to that of healthy control subjects in all battery tests. When an overall cognitive score was considered using the healthy control subjects' values, the distribution of the mean BRB-N Z score of MS patients was skewed 0.7 SD towards poorer performance. Moreover, the difference was highest $(0.85 \mathrm{SD})$ when considering only the cognitive domains that best discriminated the groups, i.e. when the abbreviated BRB-N Z score was calculated with verbal and visual memory tests and the tests of attention-executive domains. Regarding the disability scale and the BRB-N Z score, a strong correlation was established for EDSS $(r=0.540 ; p<0.01)$ and for MSFC $(r=0.456 ; p<0.01)$.

In summary, patients with MS performed worse than controls irrespectively of age, gender or education level. The degree of physical disability and disease duration significantly influenced the performance, generating poorer results in comparison to control subjects, as well as in comparison to patients with lower degrees of disability.

\section{DISCUSSION}

BRB-N is a neuropsychological screening battery of tests that is almost exclusively administered in MS trials and not yet used in the daily practice routine ${ }^{20}$. In countries where normative values have been established for BRB-N, the utility of this tool is undisputable. In a relative short period of testing, the main cognitive domains can be assessed and performances can be compared over a time frame. BRB-N has been normalized and used in Germany $^{20}$, the USA ${ }^{21-23}$, Italy ${ }^{15,24,25}$, Spain ${ }^{26,27}$, the Netherlands $^{28,29}$, Israel ${ }^{30}$, Greece ${ }^{31}$, and the United Kingdom ${ }^{32}$. In France, an adapted version of BRB-N has been proposed ${ }^{33}$.

Arnet and Forn ${ }^{34}$ have already highlighted the need for translation and application of reliable tools for assessing cognition in MS. Results from BRB-N in all of the abovementioned countries rendered similar results regarding sensitivity and specificity for detection of cognitive impairment in MS. Therefore, language does not seem to be a limiting factor for the use of this testing tool. The present study provided data regarding cognition among Brazilian patients with MS assessed by means of the BRB-N testing, after adaptation of the method in the general, healthy, young, matched pop- ulation. The time taken to perform the BRB-N testing and the possibility of doing it in routine consultations without any particular apparatus were definite advantages of this method. Testing was relatively simple, carried out by physicians during consultations and, whenever cognition was severely compromised, the patient was referred to the neuropsychologist.

\section{REFERENCES}

1. Patti F. Cognitive impairment in multiple sclerosis. Mult Scler 2009;15:2-8.

2. Messinis L, Kosmidis MH, Lyros E, Papathanasopoulos P. Assessment and rehabilitation of cognitive impairment in multiple sclerosis. Int Rev Psychiatry 2010;22:22-34.

3. Patti F, Amato MP, Trojano N, et al.; COGIMUS Study Group. Cognitive impairment and its relation with disease measures in mildly disabled patients with relapsing-remitting multiple sclerosis: baseline results from the Cognitive Impairment in Multiple Sclerosis (COGIMUS) study. Mult Scler 2009;15:779-788.

4. Kalmar JH, Gaudino EA, Moore NB, Halper J, Deluca J. The relationship between cognitive deficits and everyday functional activities in multiple sclerosis. Neuropsychology 2008;22:442-449.

5. Johnson KL, Bamer AM, Yorkston KM, Amtmann D. Use of cognitive aids and other assistive technology by individuals with multiple sclerosis. Disabil Rehabil Assist Technol 2009:4:1-8.

6. Fillippi M, Rocca MA. MRI and cognition in multiple sclerosis. Neurol Sci 2010;31(Suppl 2):S231-S234

7. Giorgio A, De Stefano N. Cognition in multiple sclerosis: relevance of lesions, brain atrophy and proton MR spectroscopy. Neurol Sci 2010; 31(Suppl 2):S245-S248.

8. Sumowski JF, Wylie GR, Chiaravalloti N, DeLuca J. Intellectual enrichment lessens the effect of brain atrophy on learning and memory in multiple sclerosis. Neurology 2010;74:1942-1945.

9. Tiemann L, Penner IK, Haupts M, Schlegel U, Calabrese P. Cognitive decline in multiple sclerosis: impact of topographic lesion distribution on differential cognitive deficit patterns. Mult Scler 2009;15:1164-1174.

10. Ferreira MLB. Cognitive deficits in multiple sclerosis: a systematic review. Arq Neuropsiquiatr 2010;68:632-641.

11. Possa MF. Neuropsychological measures in clinical practice. Neurol Sci 2010; 31 (Suppl 2):S219-S222.

12. Gainotti G. Measures of cognitive and emotional changes in multiple sclerosis and underlying models of brain dysfunction. J Neurol Sci 2006; 245:15-20.

13. Strober L, Englert J, Munchauer F, Weinstock-Guttman B, Rao S, Benedict RH. Sensitivity of conventional memory tests in multiple sclerosis: comparing the Rao Brief Repeatable Neuropsychological Battery and the Minimal Assessment of Cognitive Function in MS. Mult Scler 2009;15: 1077-1084.

14. Rao SM, Leo GJ, Bernardin L, Uverzagt F. Cognitive dysfunction in multiple sclerosis I: frequency of patterns and predictions. Neurology 1991;41: 685-691.

15. Portaccio E, Goretti B, Zipoli V, Siracusa G, Sorbi S, Amato MP. A short version of Rao's Brief Repeatable Battery as a screening tool for cognitive impairment in multiple sclerosis. Clin Neuropsychol 2009;23:268-275.

16. Bertolucci PHF, Brucki SMD, Campacci SR, Juliano Y. The mini-mental state examination in a general population: the impact of schooling. Arq Neuropsiquiatr 1994;52:1-7.

17. Zigmond AS, Snaith RP. The hospital anxiety and depression scale. Acta Psych Scand 1983;67:361-370.

18. Lublin FD, Reingold SC. Defining the clinical course of multiple sclerosis: results of an international survey. Neurology 1996;46:907-911.

19. Kurtzke JF. Rating neurologic impairment in multiple sclerosis: an expanded disability status scale (EDSS). Neurology 1983:33:1444-1452.

20. Scherer P, Baum K, Bauer H, Göhler H, Miltenburger C. Normalization of the Brief Repeatable Battery of Neuropsychological tests (BRB-N) for German-speaking regions. Nervenarzt 2004;75:984-990.

21. Bever CT Jr, Grattan L, Panitch HS, Johnson KP. The Brief Repeatable Battery of Neuropsychological Tests for Multiple Sclerosis: a preliminary serial study. Mult Scler 1995;1:165-169.

22. Strober L, Englert J, Munschauer F, Weinstock-Guttman B, Rao S, Benedict RH. Sensitivity of conventional memory tests in multiple sclerosis: com- 
paring the Rao Brief Repeatable Neuropsychological Battery and the Minimal Assessment of Cognitive Function in MS. Mult Scler 2009;15: 1077-1084.

23. Christodoulou C, Melville P, Scherl WF, et al. Perceived cognitive dysfunction and observed neuropsychological performance: Iongitudinal relation in persons with multiple sclerosis. J Int Neuropsychol Soc 2005;11: 614-619.

24. Amato MP, Portaccio E, Goretti B, et al. The Rao's Brief Repeatable Battery and Stroop Test: normative values with age, education and gender corrections in an Italian population. Mult Scler 2006;12:787-793.

25. Solari A, Mancuso L, Motta A, Mendozzi L, Serrati C. Comparison of two brief neuropsychological batteries in people with multiple sclerosis. Mult Scler 2002;8:169-176

26. Sepulcre J, Vanotti S, Hernández R, et al. Cognitive impairment in patients with multiple sclerosis using the Brief Repeatable Battery-Neuropsychology test. Mult Scler 2006;12:187-195.

27. Duque B, Sepulcre J, Bejarano B, Samaranch L, Pastor P, Villoslada P. Memory decline evolves independently of disease activity in MS. Mult Scler 2008;14:947-953.
28. Boringa JB, Lazeron $\mathrm{RH}$, Reuling IE, et al. The brief repeatable battery of neuropsychological tests: normative values allow application in multiple sclerosis clinical practice. Mult Scler 2001;7:263-267.

29. Huijbregts SC, Kalkers NF, de Sonneville LM, de Groot V, Reuling IE, Polman $\mathrm{CH}$. Differences in cognitive impairment of relapsing remitting, secondary, and primary progressive MS. Neurology 2004:63:335-339.

30. Achiron A, Barak Y. Cognitive impairment in probable multiple sclerosis. J Neurol Neurosurg Psychiatry 2003;74:443-446.

31. Potagas C, Giogkaraki E, Koutsis G, et al. Cognitive impairment in different MS subtypes and clinically isolated syndromes. J Neurol Sci 2008;267: 100-106.

32. Dent A, Lincoln NB. Screening for memory problems in multiple sclerosis. Br J Clin Psychol 2000;39:311-315.

33. Dujardin K, Sockeel P, Cabaret M, De Sèze J, Vermersch P. BCcogSEP: a French test battery evaluating cognitive functions in multiple sclerosis Rev Neurol (Paris) 2004;160:51-62.

34. Arnett P, Forn C. Neuropsychological evaluation in multiple sclerosis. Rev Neurol 2007:44:166-172. 